\title{
Active and Adaptive Services Resource Provisioning with Personalized Customization
}

\author{
Bin Wen, Ziqiang Luo \\ School of Information Science and Technology, \\ Hainan Normal University, \\ Haikou, China \\ Email: binwenwebb@gmail.com
}

\author{
Peng Liang \\ State Key Lab of Software Engineering, \\ Wuhan University, \\ Wuhan, China \\ Email: liangp@sklse.org
}

\begin{abstract}
Software as a service(SaaS),we are moving to the age of service-oriented software engineering(SOSE). But for the goal of services computing,namely on-demand service,it has not been able to achieved by far,especially the active provisioning approach for services resource.In view of these facts of services aggregation,i.e.the relative deficient services resource,single provision structure and passive selection mode for service requesters. Active provisioning of services resource with personalized customization will be focused. In this paper,we have established software architecture with personalized active custom for services resource. Customization of the active provisioning of services resource mainly includes two aspects: Firstly,for unmatched services resource of service composition, sliced or segmentation method should be chosen to acquire the individual needs of services resource according to the overall requirements.Secondly, a huge amount of legacy software will be comprehensively reused, namely servicelization.Through personalized customization of services resource,it will achieve on-demand active provisioning to furnish adequate material for dynamic services aggregation.It will also provide the production framework,process guidance and engineering support of CASE tools for services resource provisioning in runtime based on personalized customization. The feasibility and efficiency of the proposed approach are verified by a series of experiments.
\end{abstract}

Keywords-services resource provisioning; requirements engineering; ATOM; personalized customization;

\section{INTRODUCTION}

Services resource represents a sets of well-defined and interoperable Web software component. The basic physical unit will be understood as Web service, namely autonomous,open,self-describing and implementationindependent networked component [1]. The concept of services resource is from traditional software component repository that is directly related to the productivity and quality of final software product according to the degree of resource richness and meeting customer needs. Services resources provisioning means the production style, the provisioning methods and sources structure of services resource.

The importance of services resources provisioning is mainly reflected as follows : (1)Services resource that is adequate to meet the personalized needs, is material basis of services aggregation and service-based software production; (2)Effective services resource provisioning approaches should be widely used to ensure smooth implementation and efficient completion of service-based software production.

Services computing as cloud computing base and software production technologies of network era has been partially solved services representation, registration and assembly. But, the industry and academic community that is lack of considering on-demand services and related issues, i.e. the ultimate goal of computing, only focus on service discovery and composition in general. The starting point of current study assumed that services resource is sufficient, but actual development stage is not the case. Services resource that meets users' needs often does not exist or can not be run satisfactorily. As described in the literature [2]: "If no services are available for some parts, the application developer can register them in the service brokers directory and wait until the needed services are available." Thus it leads to a serious case with difficulty, complexity and low availability of services composition .

At the same time, due to the dynamic nature of Web services and error-prone services resource provisioning environment, a variety of exceptions in services resources provisioning can occur during the execution of composing services [3] . Current SOA is producer-centric, the basic idea is that service providers publish their produced services resource in order to allow consumers to search for available resources and compose its application process. But the user's preferences and needs are constantly changing, only depending on the composition of currently available resources is very difficult to build applications.

The root causes of such an awkward situation are mainly lies in :

1) The assumption that services resource rich enough in the current development stage of services computing does not hold [4]; Meanwhile, even when the rich resources to serve, the runtime services resource aimed at on-demand personalized provisioning is still important issues to solve for application building;

2) Style of resources provisioning and source organi- 
zation structure are still single [5]. Current services resource from service providers in advance of production, service consumers ( Requesters ) can only be passive to choose.

\section{Motivation}

Previously designed conceptual model with large-scale customization for networked software [1], this model also assumes that services resource is rich enough. If personalized services resource does not exist and also can not be achieved through value-added services composition, then it will relate to explore the issue of customized production which captured individual needs of service based on component resources. How to associate the back-end services resource with individual needs fragment of production model? How to embody on-demand customization requirements of service component resources and on-the-fly production? These issues are directions and concerns of this paper why focusing on active customized provisioning of services resource.

Customized services refer to supply suitable and satisfactory value of experience according to their own needs of consumers. Service-based software development is distinguished into mass customization and personalized services resource customization (Table I). The two styles complement each other to improve service quality of experience for stakeholders together.

Regarding the traditional SOA (service-oriented architecture) [6], services resource provisioning strategy is based on service providers' priority. Traditional SOA does not furnish the consumers with publishing related information functions for services production. Consumers can only retrieve, find and match provided services resource of service providers in the registry according to the needs of application, and the process is essentially a passive selection. The role and status of service consumer-centric SOA structure, which service consumer is in active position, differ from the tripartite traditional SOA technology. Service consumers not only initiated services' demand from the provided services resource to choose, match and composition service, but also can conduct personalized on-demand active customized provisioning for services.

This paper is a research about active and adaptive production approach for services resource with personalized customization. The rest of the article is organized as follows. Section III introduces the customization model of services resource. Section IV presents active customized production framework,process guidance and engineering support of CASE tools for services resource provisioning in runtime based on personalized customization, while developing prototype and obtaining empirical results. Conclusions with main contributions of proposed approach are also touched upon in Section V.

\section{SERVICES RESOURCE CUSTOMIZATION}

Definition 3.1 (Software Requirements Semantics,Req): For Req $=<S R$, Relations, Axioms $>$,SR is Software Requirements; Relations denote interrelationship among the elements of each component in SR, such as equivalent,class Property,classification etc.; Axioms represent the sets of Axiom in SR.

Definition 3.2 (Customization Requirements, CReq):

For $\mathrm{CReq}=<$ Req, Parameters $>$, Req represents requirements semantics of customized services resource, and Parameters denote parameter set of customized services resource.

Definition 3.3 (Customization of Services Resource): Given service requesters set $R=\left\{R_{1}, R_{2}, \ldots \ldots, R_{n}\right\}$ and service providers set $P=\left\{P_{1}, P_{2}, \ldots \ldots, P_{m}\right\}$, for customization requirements $C R e q_{i}$ from $R_{i} \in R(1 \leq i \leq n)$, if there exists $P_{j} \in P(1 \leq j \leq m)$ which can complete services resource production and publishing within the specified time parameters T,we call service provider $P_{j}$ in response to services resource customization of service requester $R_{i}$.

Definition 3.4 (Customized Matching Degree): For customized matching degree of services resource $C_{\text {match }}=$ SemanticMatch ${ }_{P \rightarrow R} * e^{-\frac{|t-T|}{T}}$, where SemanticMatch $_{P \rightarrow R}$ denotes semantic matching degree between the service semantics of provider's customization production and requirements semantic of requester, $t$ represents customization completion time of provider, $\mathrm{T}$ is the required customization time of requester.

According to definition 3.4, the mean value of prior services resource customized matching degree can be calculated between $\mathrm{n}$ service requestor and $\mathrm{m}$ service providers, namely:

$$
R P_{i j}=\frac{\sum_{\text {Total }}^{\text {Total }} C_{i j}}{\text { Customizeed }}
$$

$R P_{i j}$ is the current mean Customized Matching Degree of services resource for the provider $P_{j}$ in response to customized needs of the requester $R_{i}$ (Total is the total number of customization), which constitutes the services resource customization matrix $R P_{\text {Matrix }}$.

$$
R P_{\text {Matrix }}=\left(\begin{array}{ccc}
R P_{11} & \ldots & R P_{1 m} \\
\vdots & \ddots & \vdots \\
R P_{n 1} & \cdots & R P_{n m}
\end{array}\right)
$$

Definition 3.5 (Custom Preferred Provider): Given the current services resource customization matrix $R P_{\text {Matrix }}$, custom requesters and providers pair off and implement assignment operating. We call the provider the related requester's custom preferred provider if there exists a provider which corresponds to arrive at the sum of the maximum matching result.

The mathematical models for solving custom preferred provider of services resource are described as follows: 
Table I

MASS CUSTOMIZATION AND PERSONALIZED SERVICES RESOURCE CUSTOMIZATION

\begin{tabular}{|c|c|c|c|}
\hline Comparison Items & Feature/destination & $\begin{array}{l}\text { Applicable } \\
\text { stakeholders }\end{array}$ & Applicable artifacts \\
\hline Mass customization(MC) & $\begin{array}{l}\text { Large-scale productions cost and time to meet the individual needs } \\
\text { of the users; the basic idea is that the customization production will } \\
\text { transform or partially convert to mass production through restructur- } \\
\text { ing and process reengineering; mainly divided into two phases, i.e. } \\
\text { common component production and personalized assembly. }\end{array}$ & $\begin{array}{l}\text { service requester, } \\
\text { provider,software } \\
\text { architect }\end{array}$ & $\begin{array}{l}\text { final delivery of } \\
\text { products }\end{array}$ \\
\hline $\begin{array}{l}\text { personalized services resource } \\
\text { (component) customization }\end{array}$ & $\begin{array}{l}\text { Produce a wide range of services resource (components) collection to } \\
\text { adapt for individual users through personalized customization. }\end{array}$ & $\begin{array}{l}\text { service } \\
\text { requester,provider }\end{array}$ & $\begin{array}{l}\text { atomic or simple } \\
\text { composite service }\end{array}$ \\
\hline
\end{tabular}

$$
\begin{gathered}
\max C=\sum_{i=1}^{n} \sum_{j=1}^{m} R P_{i j} * x_{i j} \\
\text { s.t. }\left\{\begin{array}{c}
x_{i j}=0,1 ; i=1,2, \ldots, n, j=1,2, \ldots, m \\
\sum_{i=1}^{n} x_{i j}=1 ; j=1,2, \ldots, m \\
\sum_{j=1}^{m} x_{i j}=1 ; i=1,2, \ldots, n
\end{array}\right.
\end{gathered}
$$

This is similar to combinatorial optimization assignment problem, with reference to the Hungarian algorithm [7]. Its related conditions of the original algorithm are improved, such as the solved total minimum are transformed to maximum (The custom matching degree higher the better.). Meanwhile tag matrix is designed. The improved method is more efficient than original algorithm, easy to operate and implementation. Specific method you can see Custom Preferred Provider selection algorithm (Algorithm 1) which its time complexity is $O\left(n^{3}\right)$.

For the services resource custom provider selection, if the total custom matching degree is not required to reach a maximum just based on past selection chance to predict next selection, it is very suitable for the use of random Markov prediction method. A comprehensive forecast of the event, not only to be able to point out the incident with all possible outcomes, but also the probability of each outcome must be given with current state of the probability to predict the best choice. This could be based on historical statistical data to build a custom provider state transition probability matrix $T_{R P}$, in which rows of the matrix correspond to the requester and columns correspond to the custom provider. $P_{i j}$ denotes selection probability between requester $\mathrm{i}$ and custom $\mathrm{j}$ in current state.

$$
\begin{aligned}
& T_{R P}=\left(\begin{array}{ccc}
P_{11} & \ldots & P_{1 n} \\
\vdots & \ddots & \vdots \\
P_{n 1} & \cdots & P_{n n}
\end{array}\right) \\
& \text { s.t. }\left\{\begin{array}{c}
0 \leq P_{i j} \leq 1 \quad(i, j=1,2, \ldots, n) \\
\sum_{j=1}^{n} P_{i j}=1, \quad(i=1,2, \ldots, n)
\end{array}\right.
\end{aligned}
$$

\section{ACTIVE SERVICES RESOURCE PROVISIONING WITH PERSONALIZED CUSTOMIZATION}

Data aggregation protocol format mainly divide in RSS (Really Simple Syndication) and ATOM. RSS format emphasize on information interaction of social software, and the ATOM format is more suitable for data fusion. ATOM / RSS is a special XML file including some standard header and information items format definition. Information items contain the title, author, date, and summary etc.

\section{A. Achitecture for services resource provisioning}

Requirements Sign Ontology (RSO) [8] is semantic description of overall business process including requirementsinduced services resource (components) and control structure, and it also denotes knowledge about business workflow to guide services resource identifying and complete services aggregation.

Currently, to address the diversification and individuation of service requirements, a RGPS meta-model framework is proposed [1]. The framework includes four layers: Role layer(R), Goal layer(G), Process layer(P), and Service (S) layer. Through the associations among the four layers, the framework can be able to provide strong support for improving interoperability of requirements model. RGPSbased requirements modeling process will start from the domain analysis of problem space and complete in servicesbased solution.

On the one hand, the framework will guide how to organize domain ontology to create asset model; on the other hand, domain ontology depicts concepts and its semantic associations to support semantics for asset modeling that is characterized by ontology description (OWL or OWL-S format). It will facilitate the semantic reasoning in the SODR asset repository.

Active service technique has become the focus for services computing. Producing the services that can meet the consumer needs actively, can change passive service delivery style, which is unable to satisfy the user's requirements effectively. To this end, functions such as publishing requirements information for consumers and evaluating service quality will be added on traditional SOA. Consumers dynamically publish requirements description of services for unmatched services resource in the light of business process 


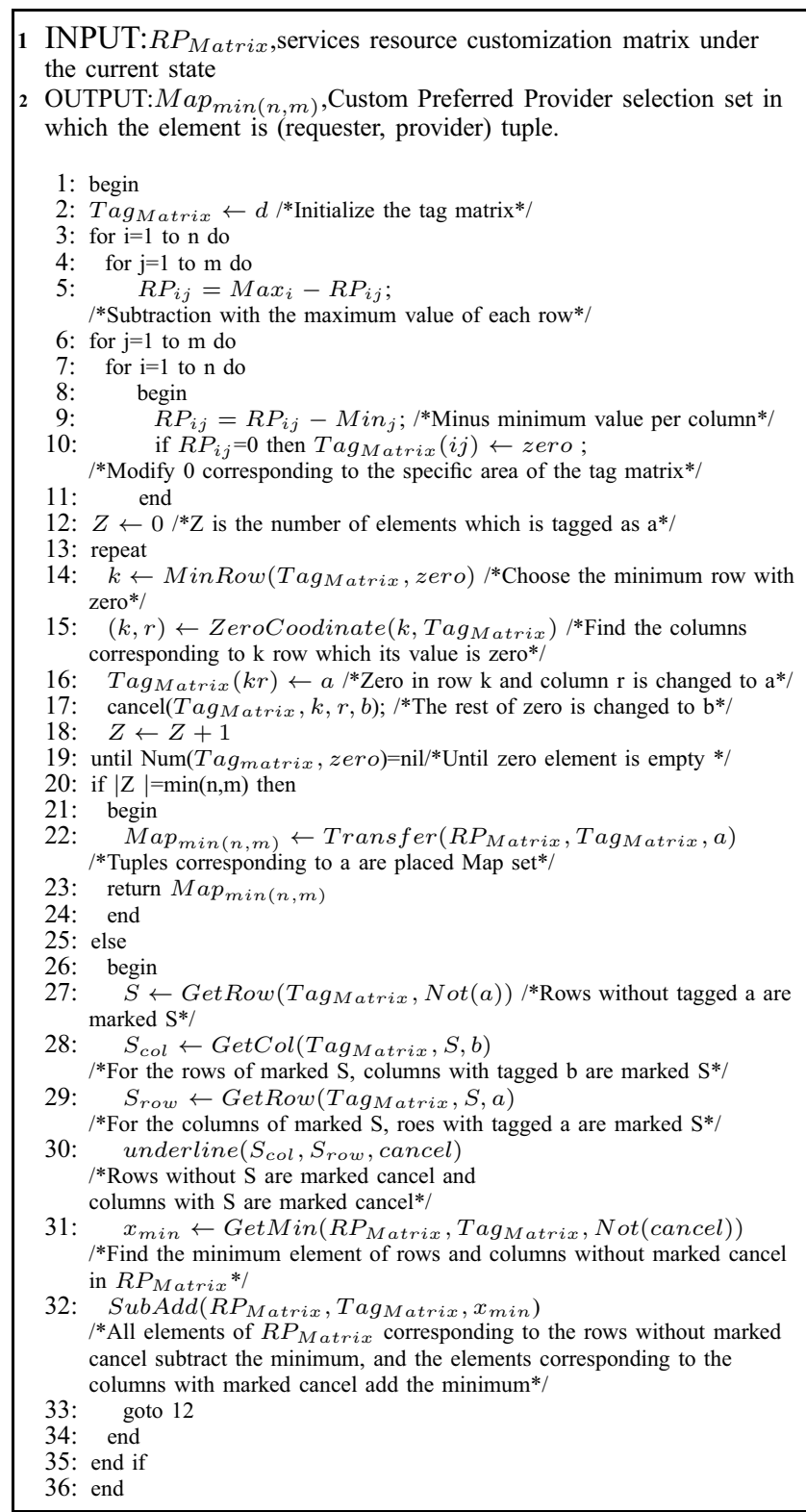

Algorithm 1: Custom Preferred Provider selection algorithm

to providers. Service providers will develop the service products in accord with the semantic description of requested services, and consumers will choose the best service to implement the business process for finishing interrupted services aggregation through quality evaluation.

Considering the feasibility and simplicity, we employ Atom format to encapsulate requirements semantics for service and fulfill subscription \& notification with service semantics by means of subscribe/inform mechanism. Solution of active service customization framework is shown in Figure 1. Service description in Atom is from service semantics in RSO that is derived from stakeholders, so

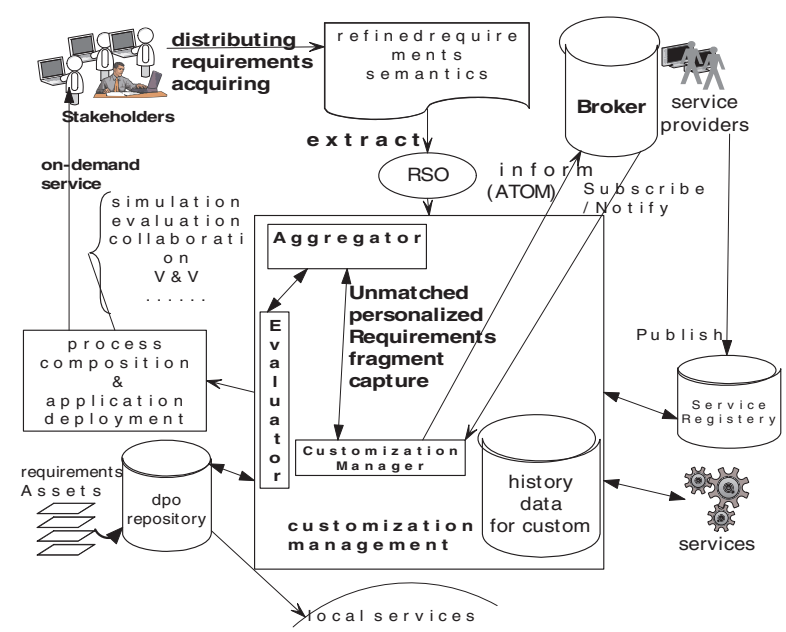

Figure 1. Consumer Requirements-driven Customization Architecture for Services Resource

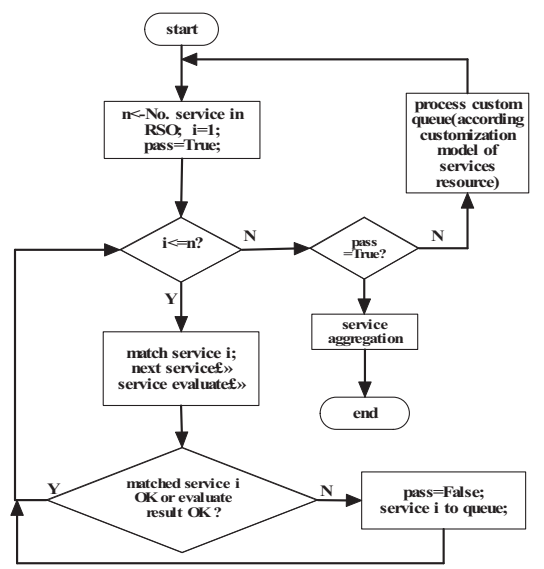

Figure 2. Services Aggregation Flowchart with Customization

customized services are also delivered actively to satisfy stakeholders.

The core part of this framework is semantic-enabled customization management platform, including customization manager, evaluator, and aggregator. Aggregator executes searching, matching, and binding the services in line with the business process of RSO. Customization manager is responsible for the customized production and management with unmatched services. Evaluator will assess and verify the quality of matched services after production.

The process of services aggregation with custom processing is shown in Figure 2. Unmatched and unqualified services will be appended into the queue of customization that will be processed to notify providers for on-demand production using Atom subscribe/inform style by customization manager. For successfully customized and matched services, they will rerun to the aggregation flow to achieve the business process. 


\section{B. Active notice of customization information}

Experimental platform mainly adopts Apache Abdera ${ }^{1}$ to generate Atom message and publishing protocol. Informed Atom format is based on the design of service semantics that is shown in Figure 3. Figure 3 shows the extension of Service segment in RGPS meta-model framework in details.

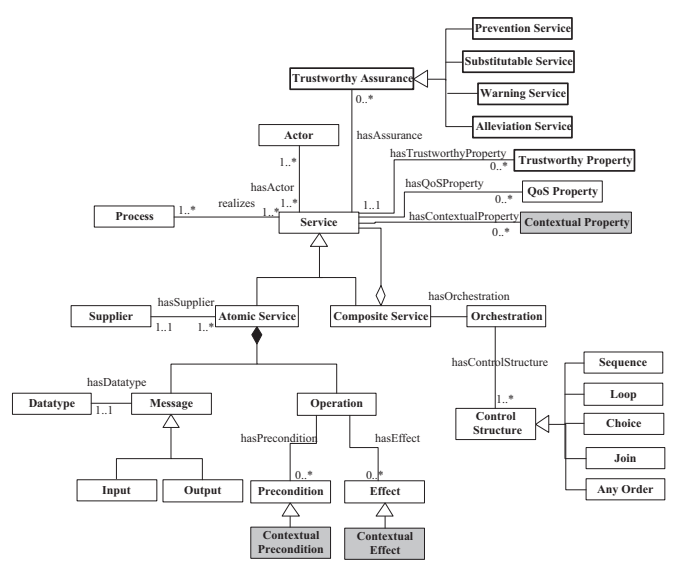

Figure 3. semantic description for service

Semantics in RSO are expressed as an OWL-S format according to the specification in Figure 3. Table II lists the corresponding relationships between every item of entry in Atom format and service semantics in RSO.

According to Table II and the semantic description of service, the description of RSO in Atom format can be finished. The transformation algorithm from semantic description of services to Atom is shown in Algorithm 2.

\section{Experiments}

1) Prototype system: Based on the Abdera library, thinfeeder ${ }^{2}$, a open source RSS reader, can be revised to display Atom and subscribe the services (see Figure 4). Also, adding 'notify' function to thinkfeeder, aggregator can get customized services data (WSDL or OWL-S file location, service endpoint URI etc.) to facilitate rerunning aggregation flow after completing the feedbacks of customized service queue.

Prototype of user requirements-driven services engineering is developed as a series of requirements-conducted services platform that have realized the main features, where requirements asset modeling for domain, service registration , requirements capture and analysis modules have launched a number of versions. The main part of prototype adopts language written in Java and PHP using Apache as a web server, MySQL database as a background. The whole system is based on the the J2EE specification to achieve distributing operations with multi-user network environment

\footnotetext{
${ }^{1}$ http://incubator.apache.org/abdera/

${ }^{2} \mathrm{http}: / /$ thinfeeder.sourceforge.net/
}

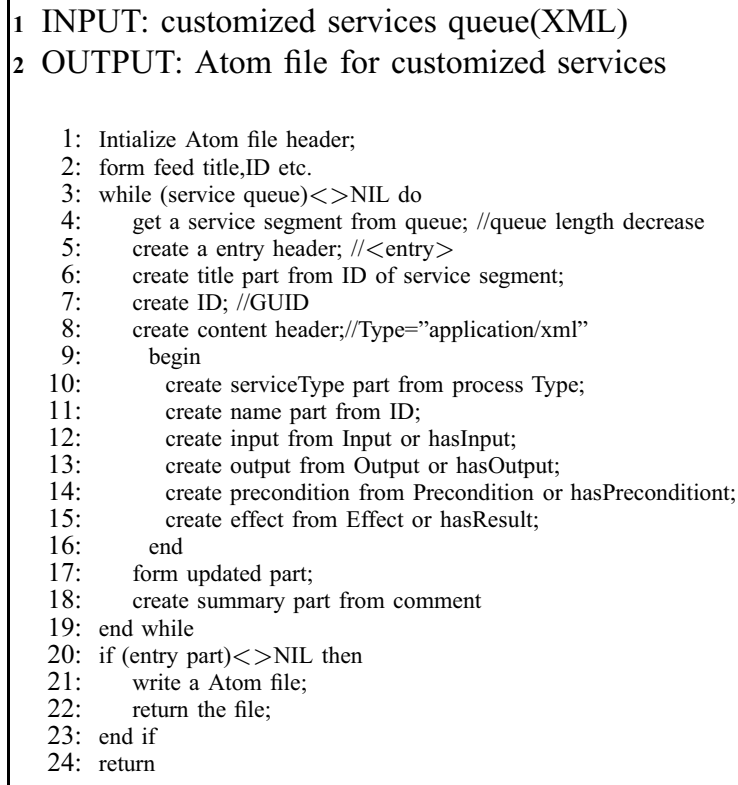

Algorithm 2: service2Atom algorithm.

and also solve the issue of heterogeneity. The platform uses a distributed service-oriented architecture as a background system architecture, and the whole system is divided into three parts , namely: requirements semantic acquisition module, service aggregation module and services resource customized modules. Among them, requirements-semantic export and service operation governance are packaged as RESTful interface to provide the modules of service aggregation and services resource customization for calling.

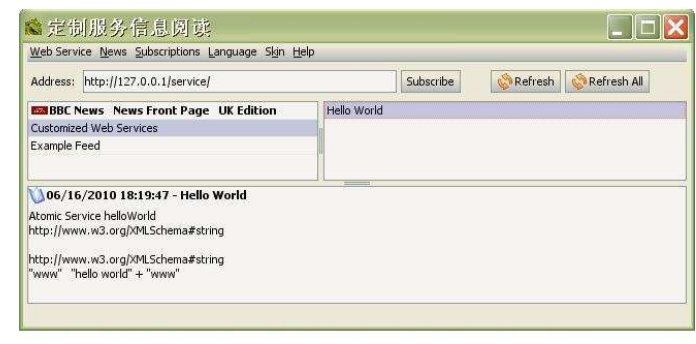

Figure 4. subscibed custom information notice for services resource

2) Experimental analysis of services resource provisioning with personalized customization: Regarding services aggregation process with customization, unmatched and unsatisfactory services resource will trigger custom platform to work according to the algorithm 1 . Resource custom program adopts JAVA through execute algorithm 2 to transfer requirements specification of services to ATOM and push to the collection of ideal service providers with custom preferred provider selection algorithm.

Custom preferred provider selection method based on 
Table II

Associations of ATOM ENTRY AND SERVICE SEMANTICS

\begin{tabular}{ll}
\hline Atom entry & Original semantics \\
\hline Atom Feed header & general information for all entry \\
AtomTitle & Human readable entry title \\
AtomId & Permanent, universally unique identifier \\
AtomUpdated & Most recent modified time for this entry \\
AtomSummary? & A short abstract, or excerpt of an entry \\
AtomSource? & Source feeds if current entry is copied from other entry \\
AtomContent? & Support the inclusion of arbitrary foreign markup \\
& \\
AtomPublished? & Initial creation or first availability of the entry \\
AtomRights? & Copyright information \\
AtomLink* & a reference from an entry to a Web resource \\
AtomContributor* & Entry contributor \\
AtomCategory* & A category associated with this entry \\
AtomAuthor* & Entry creator \\
\hline
\end{tabular}

semantics of Service customization

information generated by customization manager

service name extracted from OWL-S file

UUID generated by customization manager to identify individual web service

Most recently customized time for this service

Plain "feature" text extracted from comment segment

Not applicable for now, but can be used in the future for service composition

extending this part including serviceType (value:Atomic Service, Composite

Service), operation (input, output, precondition, effect etc.)

First published time for this service

Copyright extracted from OWL-S file

URL for OWL-S location

Not applicable for now

Arbitrary keyword or term tagged by the customization manager (if any)

Not applicable for now

Note: ? refers to the multiplicity of 0 to 1 ; ${ }^{*}$ denotes 0 to many.

custom historical data matrix compared to commonly used random selection method. Before the experiment starting, we will firstly generate customized historical data. Due to the absence of such data in industry, the data matrix will be automatically generated by the program.

Random selection uses JAVA random function and respectively compare the accuracy rate and response time (response time including historical data to generate spending). Accuracy rate $=\frac{\text { Return }}{\text { Total }}$ (Return denotes No. service providers for customized production. Total is No. service providers for pushing custom information.)

Compared measurement is the total of service providers as 10,50,100,200,250,300. Each pushed customs are $20 \%$ ordering in front of the total number of customs. The experimental operating environment consists of Dell Intel (R) Core (TM) 2 Duo CPU and 2G memory. The experimental results show that custom preferred selection algorithm according to the custom historical data significantly better than random selection for selecting accuracy rate.

\section{CONCLUSIONS}

The main contributions of this paper are summarized as follows:(1)The paper have established software architecture with personalized active custom for services resource that will select unsatisfactory requirements fragment of customers to dynamically generate custom needs in the running of the services composition. (2)By means of custom preferred provider selection approach designated to push the customization requirements, service providers complete the services resource production and customized products evaluation just in time, and the process will effectively prevent failure of services aggregation and services resource provisioning exceptions occurring.

\section{ACKNOWLEDGMENT}

This research has been supported by the Natural Science Foundation of Hainan Province(No.613162) and Higher
School Scientific Research Project of Hainan Province (No.Hjkj2013-17).

\section{REFERENCES}

[1] He Keqing, Peng Rong, Liu Wei, Networked Software. Beijing: Science Press, 2008.

[2] S. S. Yau and H. G. An, "Software engineering meets services and cloud computing," Computer, vol. 44, no. 10, pp. 46-52, 2011.

[3] Q. Z. Sheng, B. Benatallah, Z. Maamar, and A. H. Ngu, "Configurable composition and adaptive provisioning of web services," IEEE Transactions on Services Computing, vol. 2, no. 1, pp. 34-49, 2009.

[4] R. Retter, C. Fehling, D. Karastoyanova, F. Leymann, and D. Schleicher, "Combining horizontal and vertical composition of services," Service Oriented Computing and Applications (SOCA),vol. 6,no. 2, pp. 117-130, 2012.

[5] W. Tsai, B. Xiao, R. A. Paul, and Y. Chen., "Consumercentric service-oriented architecture: A new approach," in Proceedings of the Fourth IEEE Workshop on Software Technologies for Future Embedded and Ubiquitous Systems and Second International Workshop on Collaborative Computing, Integration, and Assurance (SEUS-WCCIA2006). IEEE Computer Society, 2006.

[6] Gartner, "Service oriented architectures:part 1 and part 2," http://www.gartner.com/DisplayDocument? id=302868, 1996.

[7] Hu Yunquan, Operations Research, 3rd ed. Beijing: Tsinghua University Press, 2007.

[8] Wen Bin, He Keqing, Liang Peng, Wang Jian, "Requirements Semantics-driven Aggregated Production for On-demand Service," Chinese Journal of Computers, vol. 33, no. 11, pp. 2163-2176, 2010. 